\title{
Eulerian Polynomials: from Euler's Time to the Present
}

\author{
Dominique Foata
}

\author{
Dedicated to the memory of \\ Professor Alladi Ramakrishnan
}

\begin{abstract}
The polynomials commonly called "Eulerian" today have been introduced by Euler himself in his famous book "Institutiones calculi differentialis cum eius usu in analysi finitorum ac Doctrina serierum" (chap. VII), back in 1755. They have been since thoroughly studied, extended, applied. The purpose of the present paper is to go back to Euler's memoir, find out his motivation and reproduce his derivation, surprisingly partially forgotten. The rebirth of those polynomials in a $q$-environment is due to Carlitz two centuries after Euler. A brief overview of Carlitz's method is given, as well as a short presentation of combinatorial works dealing with natural extensions of the classical Eulerian polynomials.
\end{abstract}

\section{Introduction}

Before Euler's time Jacques Bernoulli had already introduced his famous Bernoulli numbers, denoted by $B_{2 n}(n \geq 1)$ in the sequel. Those numbers can be defined by their generating function as

$$
\frac{u}{e^{u}-1}=1-\frac{u}{2}+\sum_{n \geq 1} \frac{u^{2 n}}{(2 n) !}(-1)^{n+1} B_{2 n},
$$

their first values being shown in the table:

$$
\begin{array}{c|ccccccc}
n & 1 & 2 & 3 & 4 & 5 & 6 & 7 \\
\hline B_{2 n} & 1 / 6 & 1 / 30 & 1 / 42 & 1 / 30 & 5 / 66 & 691 / 2730 & 7 / 6
\end{array}
$$

Note that besides the first term $-u / 2$ there is no term of odd rank in the series expansion (1.1), a property easy to verify. On the other hand, the factor $(-1)^{n+1}$ in formula (1.1) and the first values shown in the above table suggest that those numbers are all positive, which is true.

Jacques Bernoulli ([Be1713], p. 95-97) had introduced the numbers called after his name to evaluate the sum of the $n$-th powers of the first $m$ integers. He then proved the following summation formula

$$
\sum_{i=1}^{m} i^{n}=\frac{m^{n+1}}{n+1}+\frac{m^{n}}{2}+\frac{1}{n+1} \sum_{1 \leq r \leq n / 2}\left(\begin{array}{c}
n+1 \\
2 r
\end{array}\right) m^{n-2 r+1}(-1)^{r+1} B_{2 r}
$$

Invited address at the 10-th Annual Ulam Colloquium, University of Florida, Gainesville, February 18, 2008.

Key words and phrases. Eulerian polynomials, Bernoulli numbers, Genocchi numbers, tangent numbers, $q$-Eulerian polynomials.

Mathematics Subject Classifications. 01A50, 05A15, 05A30, 33B10. 
where $n, m \geq 1$. Once the first $\lfloor n / 2\rfloor$ Bernoulli numbers have been determined (and there are quick ways of getting them, directly derived from (1.1)), there are only $2+\lfloor n / 2\rfloor$ terms to sum on the right-hand side for evaluating $\sum_{i=1}^{m} i^{n}$, whatever the number $m$.

Euler certainly had this summation formula in mind when he looked for an expression for the alternating sum $\sum_{i=1}^{k} i^{n}(-1)^{i}$. Instead of the Bernoulli numbers he introduced another sequence $\left(G_{2 n}\right)(n \geq 1)$ of integers, later called Genocchi numbers, after the name of Peano's mentor [Ge1852]. They are related to the Bernoulli numbers by the relation

$$
G_{2 n}:=2\left(2^{2 n}-1\right) B_{2 n} \quad(n \geq 1),
$$

their first values being shown in the next table.

\begin{tabular}{c|ccccccc}
$n$ & 1 & 2 & 3 & 4 & 5 & 6 & 7 \\
\hline$B_{2 n}$ & $1 / 6$ & $1 / 30$ & $1 / 42$ & $1 / 30$ & $5 / 66$ & $691 / 2730$ & $7 / 6$ \\
\hline$G_{2 n}$ & 1 & 1 & 3 & 17 & 155 & 2073 & 38227
\end{tabular}

Of course, it is not obvious that the numbers $G_{2 n}$ defined by (1.4) are integers and furthermore odd integers. This is a consequence of the little Fermat theorem and the celebrated von Staudt-Clausen theorem (see, for instance, the classical treatise by Nielsen [Ni23] entirely devoted to the studies of Bernoulli numbers and related sequences) that asserts that the expression

$$
(-1)^{n} B_{2 n}-\sum_{p} \frac{1}{p}
$$

where the sum is over all prime numbers $p$ such that $(p-1) \mid 2 n$, is an integer. From (1.1) and (1.4) we can easily obtain the generating function for the Genocchi numbers in the form:

$$
\frac{2 u}{e^{u}+1}=u+\sum_{n \geq 1} \frac{u^{2 n}}{(2 n) !}(-1)^{n} G_{2 n} .
$$

The formula obtained by Euler for the alternating sum $\sum_{i=1}^{m} i^{n}(-1)^{i}$ is quite analogous to Bernoulli's formula (1.3). It suffices to know the first $\lfloor n / 2\rfloor$ Genocchi numbers to complete the computation. Euler's formula is the following.

Theorem 1.1. Let $\left(G_{2 n}\right)(n \geq 1)$ be the sequence of numbers defined by relation (1.4) (or by (1.6)). If $n=2 p \geq 2$, then

$$
\sum_{k=1}^{m} k^{2 p}(-1)^{k}=(-1)^{m} \frac{m^{2 p}}{2}+\sum_{k=1}^{p}\left(\begin{array}{c}
2 p \\
2 k-1
\end{array}\right)(-1)^{m+k+1} \frac{G_{2 k}}{4 k} m^{2 p-2 k+1}
$$


while, if $n=2 p+1$, the following holds:

$$
\begin{aligned}
& \sum_{k=1}^{m} k^{2 p+1}(-1)^{k}=(-1)^{m} \frac{m^{2 p+1}}{2} \\
& \quad+\sum_{k=1}^{p+1}\left(\begin{array}{c}
2 p+1 \\
2 k-1
\end{array}\right)(-1)^{m+k+1} \frac{G_{2 k}}{4 k} m^{2 p-2 k+2}+(-1)^{p+1} \frac{G_{2 p+2}}{4(p+1)}
\end{aligned}
$$

The first values of the numbers $G_{2 n}$ do appear in Euler's memoir. However, he did not bother proving that they were odd integral numbers. The two identities (1.7) and (1.8) have not become classical, in contrast to Bernoulli's formula (1.3), but the effective discovery of the Eulerian polynomials made by Euler for deriving (1.7) and (1.8) has been fundamental in numerous arithmetical and combinatorial studies in modern times. Our purpose in the sequel is to present Euler's discovery by making a contemporary reading of his calculation. Two centuries after Euler the Eulerian polynomials were given an extension in the algebra of the $q$-series, thanks to Carlitz [Ca54]. Our intention is also to discuss some aspects of that $q$-extension with a short detour to contemporary works in Combinatorics.

It is a great privilege for me to have met Professor Alladi Ramakrishnan, the brilliant Indian physicist and mathematician, who has been influential in so many fields, from Probability to Relativity Theory. He was kind enough to listen to my 2008 University of Florida Ulam Colloquium address and told me of his great admiration for Euler. I am pleased and honored therefore to dedicate the present text to his memory.

\section{Euler's definition of the Eulerian polynomials}

Let $\left(a_{i}(x)\right)(i \geq 0)$ be a sequence of polynomials in the variable $x$ and let $t$ be another variable. For each positive integer $m$ we have the banal identity:

$$
\sum_{i=0}^{m-1} a_{i}(x) t^{i}=\frac{1}{t} \sum_{i=1}^{m} a_{i-1}(x) t^{i}=a_{0}(x)+\sum_{i=1}^{m} a_{i}(x) t^{i}-a_{m}(x) t^{m} .
$$

Now, consider the operator $\Delta=\sum_{k \geq 0} \frac{(-1)^{k}}{k !} D^{k}$, where $D$ is the usual differential operator. Starting with a given polynomial $p(x)$ define

$$
\begin{aligned}
a_{i}(x) & :=\Delta^{m-i} p(x) \quad(0 \leq i \leq m) \\
\mathbf{S}(p(x), t) & :=\sum_{i=1}^{m} \Delta^{m-i} p(x) t^{i}=\sum_{i=1}^{m} a_{i}(x) t^{i} .
\end{aligned}
$$


As $D$ commutes with $\Delta$, we have $\mathbf{S}\left(D^{k} p(x), t\right)=D^{k} \mathbf{S}(p(x), t)$ for each $k \geq 0$, so that, using (2.1), we get:

$$
\begin{aligned}
a_{0}(x)+\mathbf{S}(p(x), t)-p(x) t^{m} & =a_{0}(x)+\sum_{i=1}^{m} a_{i}(x) t^{i}-a_{m}(x) t^{m} \\
& =\frac{1}{t} \sum_{i=1}^{m} a_{i-1}(x) t^{i}=\frac{1}{t} \sum_{i=1}^{m} \Delta a_{i}(x) t^{i} \\
& =\frac{1}{t} \sum_{i=1}^{m} \sum_{k \geq 0} \frac{(-1)^{k}}{k !} D^{k} a_{i}(x) t^{i} \\
& =\frac{1}{t} \sum_{k \geq 0} \frac{(-1)^{k}}{k !} \sum_{i=1}^{m} D^{k} a_{i}(x) t^{i} \\
& =\frac{1}{t} \sum_{k \geq 0} \frac{(-1)^{k}}{k !} \mathbf{S}\left(D^{k} p(x), t\right) \\
& =\frac{1}{t}\left(\mathbf{S}(p(x), t)+\sum_{k \geq 1} \frac{(-1)^{k}}{k !} \mathbf{S}\left(D^{k} p(x), t\right)\right) . \\
\text { Hence, } &
\end{aligned}
$$

$$
\mathbf{S}(p(x), t)=\frac{1}{t-1}\left(p(x) t^{m+1}-a_{0}(x) t+\sum_{k \geq 1} \frac{(-1)^{k}}{k !} \mathbf{S}\left(D^{k} p(x), t\right)\right) .
$$

We now work out specializations of (2.2) for the monomials $p(x)=x^{n}$ $(n \geq 0)$ and for $x=m$. First, we verify that:

$$
\Delta^{i} x^{n}=(x-i)^{n} \quad(0 \leq i \leq m) .
$$

It is true for $i=0$ and all $n \geq 0$. When $i \geq 0$, we have

$$
\begin{aligned}
\Delta^{i+1} x^{n} & =\Delta \Delta^{i} x^{n}=\Delta(x-i)^{n} \\
& =\sum_{k=0}^{n}(-1)^{k}\left(\begin{array}{l}
n \\
k
\end{array}\right)(x-i)^{n-k}=(x-(i+1))^{n} .
\end{aligned}
$$

Consequently,

$$
\mathbf{S}\left(x^{n}, t\right)=\sum_{i=1}^{m} \Delta^{m-i} x^{n} t^{i}=\sum_{i=1}^{m}(x-(m-i))^{n} t^{i} .
$$

Let $S\left(x^{n}, t\right):=\left.\mathbf{S}\left(x^{n}, t\right)\right|_{\{x=m\}}$, so that

$$
S\left(x^{n}, t\right)=\sum_{i=1}^{m} i^{n} t^{i}
$$

On the other hand,

$$
a_{0}(m)=\left.\Delta^{m} x^{n}\right|_{\{x=m\}}= \begin{cases}1, & \text { si } n=0 \\ 0, & \text { si } n \geq 1 .\end{cases}
$$


Identity (2.2), when $p(x)=x^{n}$ and $x=m$, becomes:

$$
\begin{aligned}
S\left(x^{n}, t\right)= & \frac{1}{t-1}\left(m^{n} t^{m+1}-a_{0}(m) t+\sum_{k=1}^{n} \frac{(-1)^{k}}{k !} S\left(D^{k} x^{n}, t\right)\right) \\
= & \frac{1}{t-1}\left(m^{n} t^{m+1}-a_{0}(m) t\right. \\
& \left.\quad+\sum_{k=1}^{n} \frac{(-1)^{k}}{k !} S\left(n(n-1) \cdots(n-k+1) x^{n-k}, t\right)\right),
\end{aligned}
$$

and, finally,

(2.6) $S\left(x^{n}, t\right)=\frac{1}{t-1}\left(m^{n} t^{m+1}-a_{0}(m) t+\sum_{k=1}^{n}(-1)^{k}\left(\begin{array}{l}n \\ k\end{array}\right) S\left(x^{n-k}, t\right)\right)$.

Another proof of identity (2.6) consists, for $m \geq 1,0 \leq k \leq m$ and $n \geq 0$, of letting $s(k, m, n):=(-1)^{k}\left(\begin{array}{l}n \\ k\end{array}\right) \sum_{i=1}^{m} i^{n-k} t^{i}$, so that $s(0, m, n)=\sum_{i=1}^{m} i^{n} t^{i}$ and of deriving the previous identity under the form

$$
s(0, m, n)=\frac{1}{t-1}\left(m^{n} t^{m+1}-a_{0}(m) t+\sum_{k=1}^{n} s(k, m, n)\right),
$$

still assuming that $a_{0}(m)=1$ if $n=0$ and 0 if $n \geq 1$. The identity is banal for $m=1$ and every $n \geq 0$. When $m \geq 2$, we have the relations

$$
s(k, m, n)=(-1)^{k}\left(\begin{array}{l}
n \\
k
\end{array}\right) m^{n-k} t^{m}+s(k, m-1, n),
$$

so that

$$
\sum_{k=1}^{n} s(k, m, n)=\left((m-1)^{n}-m^{n}\right) t^{m}+\sum_{k=1}^{n} s(k, m-1, n) .
$$

Hence, by induction on $m \geq 1$

$$
\begin{aligned}
s(0, m, n) & =\sum_{i=1}^{m} i^{n} t^{i}=m^{n} t^{m}+\sum_{i=1}^{m-1} i^{n} t^{n}=m^{n} t^{m}+s(0, m-1, n) \\
& =m^{n} t^{m}+\frac{1}{t-1}\left((m-1)^{n} t^{m}-a_{0}(m-1) t+\sum_{k=1}^{n} s(k, m-1, n)\right) \\
& =m^{n} t^{m}+\frac{1}{t-1}\left(-a_{0}(m) t+\sum_{k=1}^{n} s(k, m, n)+m^{n} t^{m}\right) \\
& =\frac{1}{t-1}\left(m^{n} t^{m+1}-a_{0}(m) t+\sum_{k=1}^{n} s(k, m, n)\right) .
\end{aligned}
$$




\section{DOMINIQUE FOATA}

As $a_{0}(m)=1$ when $n=0$, identity (2.6) yields

$$
S(1, t)=\sum_{i=1}^{m} t^{i}=\frac{1}{t-1}\left(t^{m+1}-t\right)=\frac{t\left(t^{m}-1\right)}{t-1},
$$

which is the classical formula for geometric progressions.

For discovering the polynomials, later called "Eulerian," Euler further rewrites identity (2.6) for $n=1,2,3, \ldots$ by reporting the expressions already derived for $S\left(x^{k}, t\right)(0 \leq k \leq n-1)$ into $S\left(x^{n}, t\right)$. As $a_{0}(m)=0$ for $n \geq 1$, the successive reports lead to

$$
\begin{aligned}
& S(x, t)=\frac{1}{t-1}\left(m t^{m+1}-S(1, t)\right)=\frac{1}{t-1}\left(m t^{m+1}-\frac{t\left(t^{m}-1\right)}{t-1}\right) \\
& =\frac{m t^{m+1}}{t-1} \mathbf{1}-\frac{t\left(t^{m}-1\right)}{(t-1)^{2}} \mathbf{1} ; \\
& S\left(x^{2}, t\right)=\frac{1}{t-1}\left(m^{2} t^{m+1}-2 S(x, t)+S(1, t)\right) \\
& =\frac{m^{2} t^{m+1}}{t-1} \mathbf{1}-\frac{2 m t^{m+1}}{(t-1)^{2}} \mathbf{1}+\frac{t\left(t^{m}-1\right)}{(t-1)^{3}}(\mathbf{t}+\mathbf{1}) ; \\
& S\left(x^{3}, t\right)=\frac{1}{t-1}\left(m^{3} t^{m+1}-3 S\left(x^{2}, t\right)+3 S(x, t)-S(1, t)\right) \\
& =\frac{m^{3} t^{m+1}}{t-1} \mathbf{1}-\frac{3 m^{2} t^{m+1}}{(t-1)^{2}} \mathbf{1}+\frac{3 m t^{m+1}}{(t-1)^{3}}(\mathbf{t}+\mathbf{1}) \\
& -\frac{t\left(t^{m}-1\right)}{(t-1)^{4}}\left(\mathbf{t}^{2}+\mathbf{4 t}+\mathbf{1}\right) \\
& S\left(x^{4}, t\right)=\frac{1}{t-1}\left(m^{4} t^{m+1}-4 S\left(x^{3}, t\right)+6 S\left(x^{2}, t\right)-4 S(x, t)+S(1, t)\right) \\
& =\frac{m^{4} t^{m+1}}{t-1} \mathbf{1}-\frac{4 m^{3} t^{m+1}}{(t-1)^{2}} \mathbf{1}+\frac{6 m^{2} t^{m+1}}{(t-1)^{3}}(\mathbf{t}+\mathbf{1}) \\
& -\frac{4 m t^{m+1}}{(t-1)^{4}}\left(\mathbf{t}^{2}+\mathbf{4 t}+\mathbf{1}\right)+\frac{t\left(t^{m}-1\right)}{(t-1)^{5}}\left(\mathbf{t}^{\mathbf{3}}+\mathbf{1 1} \mathbf{t}^{2}+\mathbf{1 1} \mathbf{t}+\mathbf{1}\right) ; \\
& S\left(x^{5}, t\right)=\frac{1}{t-1}\left(m^{5} t^{m+1}-5 S\left(x^{4}, t\right)+10 S\left(x^{3}, t\right)\right. \\
& \left.-10 S\left(x^{2}, t\right)+5 S(x, t)-S(1, t)\right) \\
& =\frac{m^{5} t^{m+1}}{t-1} \mathbf{1}-\frac{5 m^{4} t^{m+1}}{(t-1)^{2}} \mathbf{1}+\frac{10 m^{3} t^{m+1}}{(t-1)^{3}}(\mathbf{t}+\mathbf{1}) \\
& -\frac{10 m^{2} t^{m+1}}{(t-1)^{4}}\left(\mathbf{t}^{2}+\mathbf{4 t}+\mathbf{1}\right)+\frac{5 m t^{m+1}}{(t-1)^{5}}\left(\mathbf{t}^{\mathbf{3}}+\mathbf{1 1} \mathbf{t}^{\mathbf{2}}+\mathbf{1 1} \mathbf{t}+\mathbf{1}\right) \\
& -\frac{t\left(t^{m}-1\right)}{(t-1)^{6}}\left(\mathbf{t}^{4}+\mathbf{2 6} \mathbf{t}^{3}+\mathbf{6 6} \mathbf{t}^{2}+\mathbf{2 6} \mathbf{t}+\mathbf{1}\right) \text {. }
\end{aligned}
$$


Let $A_{n}(t)$ be the coefficient of $\frac{t\left(t^{m}-1\right)}{(t-1)^{n+1}}$ in the above expansions of $S\left(x^{n}, t\right)$ for $n=0,1,2,3,4,5$, so that $A_{0}(t)=A_{1}(t)=1, A_{2}(t)=t+1$, $A_{3}(t)=t^{2}+4 t+1, A_{4}(t)=t^{3}+11 t^{2}+11 t+1, A_{5}(t)=t^{4}+26 t^{3}+66 t^{2}+26 t+1$. We observe that the expression of $A_{n}(t)$ in the expansion of $S\left(x^{n}, t\right)$ is obtained by means of the formula

$$
A_{n}(t)=\sum_{k=0}^{n-1}\left(\begin{array}{l}
n \\
k
\end{array}\right) A_{k}(t)(t-1)^{n-1-k} .
$$

We also see that $S\left(x^{n}, t\right)=\sum_{i=1}^{m} i^{n} t^{i}$ can be expressed as

$$
S\left(x^{n}, t\right)=\sum_{l=1}^{n}(-1)^{n+l}\left(\begin{array}{c}
n \\
l
\end{array}\right) \frac{t^{m+1} A_{n-l}(t)}{(t-1)^{n-l+1}} m^{l}+(-1)^{n} \frac{t\left(t^{m}-1\right)}{(t-1)^{n+1}} A_{n}(t) .
$$

Once those two facts have been observed, it remains to prove that the new expression found for $S\left(x^{n}, t\right)$ holds for every $n \geq 0$, as stated next.

Theorem 2.1. Let $A_{0}(t):=1$ and let $\left(A_{n}(t)\right)(n \geq 0)$ be the sequence of polynomials inductively defined by (2.7). Then identity (2.8) holds.

Proof. For proving such a theorem today we proceed by induction, reporting (2.7) into (2.6) and making an appropriate change of variables in the finite sums. This is the first proof that is now presented. At the time of Euler the " $\sum$ " notation does not exist, and of course not the double sums! For example, (2.6) is displayed as:

$$
\begin{aligned}
S . x^{n} p^{x}=\frac{1}{p-1}\left(x^{n} p^{x+1}\right. & -A^{p}-n S \cdot x^{n-1} p^{x}+\frac{n(n-1)}{1 \cdot 2} S \cdot x^{n-2} p^{x} \\
& -\frac{n(n-1)(n-2)}{1 \cdot 2 \cdot 3} S \cdot x^{n-3} p^{x} \\
& \left.+\frac{n(n-1)(n-2)(n-3)}{1 \cdot 2 \cdot 3 \cdot 4} S \cdot x^{n-4} p^{x}-\& c .\right) .
\end{aligned}
$$

Euler is then led to imagine another proof based in fact on the concept of linear independence.

For the first proof start with identity (2.6) and apply the induction hypothesis to all the terms $S\left(x^{n-k}, t\right)$ of the sum, with $k$ running from 1 to $n$ :

$$
S\left(x^{n}, t\right)=\frac{1}{t-1}\left(m^{n} t^{m+1}+\sum_{k=1}^{n}(-1)^{k}\left(\begin{array}{l}
n \\
k
\end{array}\right) S\left(x^{n-k}, t\right)\right)
$$




\section{DOMINIQUE FOATA}

$$
\begin{aligned}
& =\frac{1}{t-1}\left(m^{n} t^{m+1}+\sum_{j=0}^{n-1}(-1)^{n-j}\left(\begin{array}{l}
n \\
j
\end{array}\right) S\left(x^{j}, t\right)\right) \\
& =\frac{1}{t-1}\left(m^{n} t^{m+1}+\sum_{j=0}^{n-1}(-1)^{n-j}\left(\begin{array}{c}
n \\
j
\end{array}\right)\right. \\
& \left.\quad \sum_{l=1}^{j}(-1)^{j+l}\left(\begin{array}{l}
j \\
l
\end{array}\right) \frac{t^{m+1} A_{j-l}(t)}{(t-1)^{j-l+1}} m^{l}+(-1)^{j} \frac{t\left(t^{m}-1\right)}{(t-1)^{j+1}} A_{j}(t)\right) \\
& =\frac{m^{n} t^{m+1}}{t-1}+\sum_{l=1}^{n-1} \sum_{j=l}^{n-1}(-1)^{n+l} \frac{n !}{(n-j) ! l !(j-l) !} \frac{t^{m+1} A_{j-l}(t)}{(t-1)^{j-l+2}} m^{l} \\
& \quad+(-1)^{n} \frac{t\left(t^{m}-1\right)}{(t-1)^{n+1}} \sum_{j=0}^{n-1}\left(\begin{array}{c}
n \\
j
\end{array}\right) A_{j}(t)(t-1)^{n-j-1} .
\end{aligned}
$$

With $j=k-l$ we deduce

$$
\begin{aligned}
& S\left(x^{n}, t\right)=\frac{m^{n} t^{m+1}}{t-1}+\sum_{l=1}^{n-1}(-1)^{n+l}\left(\begin{array}{l}
n \\
l
\end{array}\right) t^{m+1} m^{l} \sum_{k=0}^{n-1-l}\left(\begin{array}{c}
n-l \\
k
\end{array}\right) \frac{A_{k}(t)}{(t-1)^{k+2}} \\
& +(-1)^{n} \frac{t\left(t^{m}-1\right)}{(t-1)^{n+1}} A_{n}(t) \\
& =\frac{m^{n} t^{m+1}}{t-1}+\sum_{l=1}^{n-1}(-1)^{n+l}\left(\begin{array}{l}
n \\
l
\end{array}\right) \frac{t^{m+1} m^{l}}{(t-1)^{n-l+1}} \\
& \sum_{k=0}^{n-1-l}\left(\begin{array}{c}
n-l \\
k
\end{array}\right) A_{k}(t)(t-1)^{n-l-k-1}+(-1)^{n} \frac{t\left(t^{m}-1\right)}{(t-1)^{n+1}} A_{n}(t) \\
& =\frac{m^{n} t^{m+1}}{t-1}+\sum_{l=1}^{n-1}(-1)^{n+l}\left(\begin{array}{l}
n \\
l
\end{array}\right) \frac{t^{m+1} m^{l}}{(t-1)^{n-l+1}} A_{n-l}(t) \\
& +(-1)^{n} \frac{t\left(t^{m}-1\right)}{(t-1)^{n+1}} A_{n}(t) \\
& =\sum_{l=1}^{n}(-1)^{n+l}\left(\begin{array}{l}
n \\
l
\end{array}\right) \frac{t^{m+1} m^{l}}{(t-1)^{n-l+1}} A_{n-l}(t)+(-1)^{n} \frac{t\left(t^{m}-1\right)}{(t-1)^{n+1}} A_{n}(t) .
\end{aligned}
$$

The original proof by Euler can be reproduced as follows. With $p(x)=x^{n}$ $(n \geq 1)$ and $l=0,1, \ldots, n$ let

$$
\begin{aligned}
& Y_{l}:=S\left(D^{l} p(x), t\right) \\
& Z_{l}:= \begin{cases}\frac{D^{l} p(m)}{t-1} t^{m+1}, & \text { si } 0 \leq l \leq n-1 ; \\
\frac{D^{n} p(m)}{t-1}\left(t^{m+1}-t\right), & \text { si } l=n .\end{cases}
\end{aligned}
$$


In formula (2.2) successively replace $p(x)$ by $D^{l} p(x)$ for $l=0,1, \ldots, n$. We obtain

$$
Y_{l}=Z_{l}+\sum_{j=1}^{n-l} \frac{(-1)^{j}}{j !} \frac{1}{t-1} Y_{l+j} \quad(0 \leq l \leq n),
$$

remembering that $a_{0}(x)$ is null for $x=m$, and 1 for $Y_{n}=S\left(D^{n} p(x), t\right)$. Now, each of the two sequences $\left(Y_{0}, Y_{1}, \ldots, Y_{n}\right),\left(Z_{0}, Z_{1}, \ldots, Z_{n}\right)$ is a basis for the algebra of polynomials of degree at most equal to $n$, since both $Y_{l}$ and $Z_{l}$ are polynomials of degree $n-l(l=0,1, \ldots, n)$. Accordingly, there exists a sequence $\left(b_{0}, b_{1}, \ldots, b_{n}\right)$ of coefficients such that

$$
Y_{0}=\sum_{l=0}^{n} b_{l}(-1)^{l} Z_{l}
$$

so that, by using (2.9),

$$
\begin{aligned}
Y_{0} & =\sum_{l=0}^{n} b_{l}(-1)^{l}\left(Y_{l}-\sum_{j=1}^{n-l} \frac{(-1)^{j}}{j !} \frac{1}{t-1} Y_{l+j}\right) \\
& =\sum_{k=0}^{n}\left(b_{k}(-1)^{k}-\sum_{\substack{l+j=k, l \geq 0, j \geq 1}} b_{l}(-1)^{l} \frac{(-1)^{j}}{j !} \frac{1}{t-1}\right) Y_{k} .
\end{aligned}
$$

Hence, $b_{0}=1$; furthermore, for $k=1,2, \ldots, n$,

$$
b_{k}=\frac{1}{t-1}\left(\frac{b_{k-1}}{1 !}+\frac{b_{k-2}}{2 !}+\cdots+\frac{b_{1}}{(k-1) !}+\frac{b_{0}}{k !}\right)
$$

or still

$$
k !(t-1)^{k} b_{k}=\sum_{l=0}^{l-1}\left(\begin{array}{l}
k \\
l
\end{array}\right) l !(t-1)^{l} b_{l}(t-1)^{k-1-l} .
$$

By comparison with the induction formula for the Eulerian polynomials

$$
b_{n}=\frac{1}{n !(t-1)^{n}} A_{n}(t) \quad(n \geq 0) .
$$

Finally, reporting $b_{n}$ into (2.10) yields (2.8).

\section{A formulary for the Eulerian polynomials}

The polynomials $A_{n}(t)(n=0,1, \ldots)$, inductively defined by $A_{0}(t):=1$ and identity (2.7) for $n \geq 1$ are unanimously called Eulerian polynomials. It is hard to trace back the exact origin of their christening. 
Form the exponential generating function

$$
A(t, u):=\sum_{n \geq 0} A_{n}(t) \frac{u^{n}}{n !} .
$$

Then (2.7) is equivalent to the identity

$$
\begin{aligned}
A(t, u) & =1+\sum_{n \geq 1} \sum_{\substack{k+l=n \\
0 \leq k \leq n-1}} A_{k}(t) \frac{u^{k}}{k !} \frac{(t-1)^{l-1}}{l !} u^{l} \\
& =1+A(t, u) \times \sum_{l \geq 1} \frac{(t-1)^{l-1}}{l !} u^{l} \\
& =1+A(t, u) \frac{1}{t-1}(\exp (u(t-1))-1)
\end{aligned}
$$

hence

$$
A(t, u)=\sum_{n \geq 0} A_{n}(t) \frac{u^{n}}{n !}=\frac{t-1}{t-\exp (u(t-1))}
$$

which is the traditional exponential generating function for the Eulerian polynomials, explicitly given by Euler himself in his memoir.

We may also write:

$$
\begin{aligned}
\sum_{n \geq 0} \frac{A_{n}(t)}{(1-t)^{n+1}} \frac{u^{n}}{n !} & =\frac{e^{u}}{1-t e^{u}}=e^{u} \sum_{j \geq 0}\left(t e^{u}\right)^{j} \\
& =\sum_{j \geq 0} t^{j} \sum_{n \geq 0} \frac{(u(j+1))^{n}}{n !}=\sum_{n \geq 0} \frac{u}{n !} \sum_{j \geq 0} t^{j}(j+1)^{n},
\end{aligned}
$$

which leads to another equivalent definition of the Eulerian polynomials

$$
\frac{A_{n}(t)}{(1-t)^{n+1}}=\sum_{j \geq 0} t^{j}(j+1)^{n} \quad(n \geq 0)
$$

which is the most common starting definition of those polynomials today.

Now rewrite identity (2.8) as

$$
\sum_{i=1}^{m} i^{n} t^{i}=-t^{m+1} \sum_{k=0}^{n}\left(\begin{array}{l}
n \\
k
\end{array}\right) \frac{A_{k}(t)}{(1-t)^{k+1}} m^{n-k}+\frac{t A_{n}(t)}{(1-t)^{n+1}} .
$$

A simple argument on the order of the formal series in $t$ shows that, when $m$ tends to infinity, (3.3) implies (3.2). Conversely, replace each fraction 
$A_{k}(t) /(1-t)^{k+1}$ on the right-hand side of (3.3) by $\sum_{j \geq 0} t^{j}(j+1)^{k}$, which is the right-hand side of (3.2). An easy calculation shows that the right-hand side of (3.3) becomes

$$
-\sum_{j \geq 0} t^{m+1+j}(m+1+j)^{n}+t \sum_{j \geq 0} t^{j}(j+1)^{n}=\sum_{i=1}^{m} i^{n} t^{i} .
$$

Consequently, identity (3.2) and its finite form (3.3) are also equivalent.

The relation

$$
A_{n}(t)=(1+(n-1) t) A_{n-1}(t)+t(1-t) \cdot D A_{n-1}(t) \quad(n \geq 1),
$$

where $D$ stands for the differential operator in $t$, can be proved from (3.2) as follows: the right-hand side of (3.4) is equal to:

$$
\begin{array}{r}
(1-t)^{n} \sum_{j \geq 0} t^{j}(j+1)^{n-1}(1+(n-1) t-n t+(1-t) j) \\
=(1-t)^{n} \sum_{j \geq 0} t^{j}(j+1)^{n-1}(1-t)(j+1) \\
=(1-t)^{n+1} \sum_{j \geq 0} t^{j}(j+1)^{n}=A_{n}(t) .
\end{array}
$$

Finally, let

$$
A_{n}(t):=\sum_{k \geq 0} A_{n, k} t^{k}
$$

By (3.2) $A_{0}(t)=(1-t) /(1-t)=1$ and the constant coefficient of each polynomial $A_{n}(t)$ is $A_{n, 0}=1$. In (3.4) the coefficient of $t^{k}(k \geq 1)$ is equal to $A_{n, k}$ on the left and $A_{n-1, k}+(n-1) A_{n-1, k-1}+k A_{n-1, k}-(k-1) A_{n-1, k-1}$ on the right. As $A_{n, k}=0$ for $k \geq n \geq 1$, we obtain the recurrence relation

$$
\begin{gathered}
A_{n, k}=(k+1) A_{n-1, k}+(n-k) A_{n-1, k-1} \quad(1 \leq k \leq n-1) ; \\
A_{n, 0}=1 \quad(n \geq 0) ; \quad A_{n, k}=0 \quad(k \geq n),
\end{gathered}
$$

so that each $A_{n}(t)$ is a polynomial with positive integral coefficients. The first values of the coefficients $A_{n, k}$, called Eulerian numbers, are shown in the next table.

\begin{tabular}{|r|ccccccc|}
\hline $\mathrm{k}=$ & 0 & 1 & 2 & 3 & 4 & 5 & 6 \\
\hline $\mathrm{n}=1$ & 1 & & & & & & \\
2 & 1 & 1 & & & & & \\
3 & 1 & 4 & 1 & & & & \\
4 & 1 & 11 & 11 & 1 & & & \\
5 & 1 & 26 & 66 & 26 & 1 & & \\
6 & 1 & 57 & 302 & 302 & 57 & 1 & \\
7 & 1 & 120 & 1191 & 2416 & 1191 & 120 & 1 \\
\hline
\end{tabular}


In summary, the Eulerian polynomials $A_{n}(t)=\sum_{k=0}^{n} A_{n, k} t^{k}(n \geq 0)$ are
fined by the relations:

$$
\begin{aligned}
& A_{0}(t)=1 ; \quad A_{n}(t)=\sum_{k=0}^{n-1}\left(\begin{array}{l}
n \\
k
\end{array}\right) A_{k}(t)(t-1)^{n-1-k}(n \geq 1) \\
& \sum_{i=1}^{m} i^{n} t^{i}=\sum_{l=1}^{n}(-1)^{n+l}\left(\begin{array}{l}
n \\
l
\end{array}\right) \frac{t^{m+1} A_{n-l}(t)}{(t-1)^{n-l+1}} m^{l}+(-1)^{n} \frac{t\left(t^{m}-1\right)}{(t-1)^{n+1}} A_{n}(t), \\
& \text { for } m \geq 1, n \geq 0, \text { as previously mentioned, but also by: } \\
& \sum_{n \geq 0} A_{n}(t) \frac{u^{n}}{n !}=\frac{t-1}{t-\exp (u(t-1))} \\
& \frac{A_{n}(t)}{(1-t)^{n+1}}=\sum_{j \geq 0} t^{j}(j+1)^{n} \quad(n \geq 0) ; \\
& A_{0}(t)=1, A_{n}(t)=(1+(n-1) t) A_{n-1}(t)+t(1-t) \cdot D A_{n-1}(t)(n \geq 1) \\
& A_{n, k}=(k+1) A_{n-1, k}+(n-k) A_{n-1, k-1} \quad(1 \leq k \leq n-1) \\
& A_{n, 0}=1 \quad(n \geq 0) ; \quad A_{n, k}=0 \quad(k \geq n) ; \\
& A_{n, k}=\sum_{0 \leq i \leq k}(-1)^{i}(k-i+1)^{n}\left(\begin{array}{c}
n+1 \\
i
\end{array}\right) \quad(0 \leq k \leq n-1) \\
& x^{n}=\sum_{0 \leq k \leq n-1}\left(\begin{array}{c}
x+k \\
n
\end{array}\right) A_{n, k} \quad(n \geq 0) .
\end{aligned}
$$

The last two relations are easy to establish. For the first one make use of (3.2), starting with $A_{n}(t)(1-t)^{-(n+1)}$. For the second, called Worpitzky identity, simply calculate the coefficient of $t^{k}$ in $(1-t)^{n+1} \sum_{n>0} t^{n}(j+1)^{n}$. As mentioned earlier, the first three relations (2.7), (2.8) and (3.1) are due to Euler. The fourth relation (3.2) must also be attributed to him, as he used it for $t=-1$ in a second memoir [Eul1768] to give a sense to the divergent series $\sum_{j}(-1)^{j}(j+1)^{m}$. Note that $(2.8)$ is fundamental for the next calculation involving tangent numbers. It seems to have been forgotten in today's studies.

\section{A relation with the tangent numbers}

The tangent numbers $T_{2 n-1}(n \geq 1)$ are defined as the coefficients of the Taylor expansion of $\tan u$ :

$$
\begin{aligned}
\tan u & =\sum_{n \geq 1} \frac{u^{2 n-1}}{(2 n-1) !} T_{2 n-1} \\
& =\frac{u}{1 !} 1+\frac{u^{3}}{3 !} 2+\frac{u^{5}}{5 !} 16+\frac{u^{7}}{7 !} 272+\frac{u^{9}}{9 !} 7936+\frac{u^{11}}{11 !} 353792+\cdots
\end{aligned}
$$

But, starting with (1.6), the generating function for the Genocchi numbers 
$G_{2 n}(n \geq 1)$ can be evaluated as follows:

$$
\begin{aligned}
\sum_{n \geq 1} \frac{u^{2 n}}{(2 n) !} G_{2 n} & =\sum_{n \geq 1} \frac{(i u)^{2 n}}{(2 n) !}(-1)^{n} G_{2 n} \\
& =\frac{2 i u}{e^{i u}+1}-i u=\frac{i u\left(1-e^{i u}\right)}{1+e^{i u}}=u \tan (u / 2)
\end{aligned}
$$

Hence,

$$
\sum_{n \geq 1} \frac{u^{2 n}}{(2 n) !} G_{2 n}=u \tan (u / 2)=\sum_{n \geq 1} \frac{u^{2 n}}{2^{2 n-1}(2 n-1) !} T_{2 n-1},
$$

and then

$$
n T_{2 n-1}=2^{2 n-2} G_{2 n}=2^{2 n-1}\left(2^{2 n}-1\right) B_{2 n} \quad(n \geq 1) .
$$

The first values of the tangent numbers $T_{2 n-1}(n \geq 1)$, compared with the Genocchi numbers, are shown in the next table.

\begin{tabular}{c|ccccccc}
$n$ & 1 & 2 & 3 & 4 & 5 & 6 & 7 \\
\hline$G_{2 n}$ & 1 & 1 & 3 & 17 & 155 & 2073 & 38227 \\
\hline$T_{2 n-1}$ & 1 & 2 & 16 & 272 & 7936 & 353.792 & 22.368 .256
\end{tabular}

In the exponential generating function (3.1) for the Eulerian polynomials $A_{n}(t)$ replace $t$ by -1 and $u$ by $i u$ with $i=\sqrt{-1}$. We get:

Hence

$$
\sum_{n \geq 0} A_{n}(-1) \frac{(i u)^{n}}{n !}=\frac{2}{1+e^{-2 i u}} .
$$

$$
\begin{aligned}
\sum_{n \geq 1} i^{n-1} A_{n}(-1) \frac{u^{n}}{n !} & =\frac{1}{i}\left(\frac{2}{1+e^{-2 i u}}-1\right)=\frac{1}{i} \frac{1-e^{-2 i u}}{1+e^{-2 i u}}=\tan u \\
& =\sum_{n \geq 1} T_{2 n-1} \frac{u^{2 n-1}}{(2 n-1) !} .
\end{aligned}
$$

Accordingly,

$$
A_{2 n}(-1)=0, \quad A_{2 n-1}(-1)=(-1)^{n-1} T_{2 n-1} \quad(n \geq 1) .
$$

With $t=-1$ identity (2.8) becomes

$$
\sum_{k=1}^{m} k^{n}(-1)^{k}=(-1)^{m} \sum_{k=0}^{n}\left(\begin{array}{l}
n \\
k
\end{array}\right) \frac{A_{k}(-1)}{2^{k+1}} m^{n-k}+\frac{(-1) A_{n}(-1)}{2^{n+1}} .
$$

When $n=2 p \geq 2$ we get:

$$
\sum_{k=1}^{m} k^{2 p}(-1)^{k}=(-1)^{m+1} \frac{m^{2 p}}{2}+\sum_{k=1}^{p}\left(\begin{array}{c}
2 p \\
2 k-1
\end{array}\right)(-1)^{m+k-1} \frac{T_{2 k-1}}{2^{2 k}} m^{2 p-2 k+1}
$$


Using the relation $n T_{2 n-1}=2^{2 n-2} G_{2 n}(n \geq 1)$ this can be rewritten in terms of the Genocchi numbers as:

$$
\sum_{k=1}^{m} k^{2 p}(-1)^{k}=(-1)^{m} \frac{m^{2 p}}{2}+\sum_{k=1}^{p}\left(\begin{array}{c}
2 p \\
2 k-1
\end{array}\right)(-1)^{m+k+1} \frac{G_{2 k}}{4 k} m^{2 p-2 k+1}
$$

When $n=2 p+1 \geq 1$ we get:

$$
\begin{aligned}
& \sum_{k=1}^{m} k^{2 p+1}(-1)^{k} \\
& \quad=(-1)^{m+1} \frac{m^{2 p+1}}{2}+\sum_{k=1}^{p+1}\left(\begin{array}{c}
2 p+1 \\
2 k-1
\end{array}\right)(-1)^{m+k} \frac{T_{2 k-1}}{2^{2 k}} m^{2 p-2 k+2} \\
& +(-1)^{p+1} \frac{T_{2 p+1}}{2^{2 p+2}}
\end{aligned}
$$

so that in terms of the Genocchi numbers

$$
\begin{aligned}
& \sum_{k=1}^{m} k^{2 p+1}(-1)^{k} \\
& =(-1)^{m} \frac{m^{2 p+1}}{2}+\sum_{k=1}^{p+1}\left(\begin{array}{c}
2 p+1 \\
2 k-1
\end{array}\right)(-1)^{m+k+1} \frac{G_{2 k}}{4 k} m^{2 p-2 k+2} \\
& +(-1)^{p+1} \frac{G_{2 p+2}}{4(p+1)}
\end{aligned}
$$

Both identities (4.4) and (4.6) were established by Euler. This achieves the proof of Theorem 1.1.

\section{The Carlitz $q$-Eulerian polynomials}

Recall the traditional $q$-ascending factorial defined for each ring element $\omega$ and each variable $q$ by

$$
\begin{aligned}
(\omega ; q)_{k} & := \begin{cases}1, & \text { if } k=0 \\
(1-\omega)(1-\omega q) \cdots\left(1-\omega q^{k-1}\right), & \text { if } k \geq 1\end{cases} \\
(\omega ; q)_{\infty} & :=\prod_{k \geq 0}\left(1-\omega q^{k}\right)
\end{aligned}
$$

the $q$-binomial coefficients

$$
\left[\begin{array}{l}
n \\
k
\end{array}\right]_{q}:=\frac{(q ; q)_{n}}{(q ; q)_{k}(q ; q)_{n-k}} \quad(0 \leq k \leq n)
$$

and the $q$-analogs of integers and factorials

$$
\begin{aligned}
{[n]_{q} } & :=\frac{(1-q)^{n}}{(1-q)^{n}}=1+q+q^{2}+\cdots+q^{n-1} \\
{[n] !_{q} } & :=[n]_{q}[n-1]_{q} \cdots[1]_{q} .
\end{aligned}
$$


As $\lim _{q \rightarrow 1}(t ; q)_{n+1}=(1-t)^{n+1}$ and $\lim _{q \rightarrow 1}[j+1]_{q}=j+1$, definition (3.2) suggests that a new sequence of polynomials $A_{n}(t, q)$, called the $q$-Eulerian polynomials, can be defined by the identity

$$
\frac{A_{n}(t, q)}{(t ; q)_{n+1}}=\sum_{j \geq 0} t^{j}\left([j+1]_{q}\right)^{n} \quad(n \geq 0),
$$

as was done by Carlitz [Ca54] in his seminal paper, thereby entering the $q$-series environment initiated by Heine [He1847]. Also see Gasper and Rahman [GR90].

By analogy with the Eulerian polynomials we can replace the infinite series (5.1) by a finite sum and try to express the left-hand side as a linear combination of fractions $A_{k}(t, q) /(t ; q)_{k+1}(0 \leq k \leq n)$. From (5.1) we get:

$$
\begin{aligned}
& \sum_{j=1}^{m} t^{j}\left([j]_{q}\right)^{n}=\sum_{k=0}^{m-1} t^{k+1}\left([k+1]_{q}\right)^{n} \\
& \quad=t \sum_{j \geq 0} t^{j}\left([j+1]_{q}\right)^{n}-\sum_{j \geq 0} t^{m+1+j}\left([m+1+j]_{q}\right)^{n} \\
& \quad=t \frac{A_{n}(t, q)}{(t ; q)_{n+1}}-t^{m+1} \sum_{j \geq 0} t^{j}\left(1+q+\cdots+q^{j}+q^{j+1}+\cdots+q^{j+m}\right)^{n} \\
& =t \frac{A_{n}(t, q)}{(t ; q)_{n+1}}-t^{m+1} \sum_{j \geq 0} t^{j} \sum_{k=0}^{n}\left(\begin{array}{l}
n \\
k
\end{array}\right)\left(1+q+\cdots+q^{j}\right)^{k}\left(q^{j+1}+\cdots+q^{j+m}\right)^{n-k} \\
& =t \frac{A_{n}(t, q)}{(t ; q)_{n+1}}-t^{m+1} \sum_{j \geq 0} t^{j} \sum_{k=0}^{n}\left(\begin{array}{l}
n \\
k
\end{array}\right)[j+1]_{q}^{k} q^{(j+1)(n-k)}[m]_{q}^{n-k} \\
& =t \frac{A_{n}(t, q)}{(t ; q)_{n+1}}-t^{m+1} \sum_{k=0}^{n}\left(\begin{array}{l}
n \\
k
\end{array}\right) q^{n-k}[m]_{q}^{n-k} \sum_{j \geq 0}\left(t q^{(n-k)}\right)^{j}[j+1]_{q}^{k} .
\end{aligned}
$$

This establishes the identity

$$
\sum_{j=1}^{m} t^{j}\left([j]_{q}\right)^{n}=t \frac{A_{n}(t, q)}{(t ; q)_{n+1}}-t^{m+1} \sum_{k=0}^{n}\left(\begin{array}{l}
n \\
k
\end{array}\right) q^{n-k}[m]_{q}^{n-k} \frac{A_{k}\left(t q^{n-k}, q\right)}{\left(t q^{n-k} ; q\right)_{k+1}}
$$

apparently (?) new, that $q$-generalizes (2.8). Naturally, both definitions (5.1) and (5.2) for the polynomials $A_{n}(t, q)$ are equivalent.

Other equivalent definitions can be derived as follows. Starting with (5.1) we can express $A_{n}(t, q)$ as a polynomial in $t$ as follows;

$$
\frac{A_{n}(t, q)}{(t ; q)_{n+1}}=\sum_{j \geq 0} t^{j}\left([j+1]_{q}\right)^{n}=\sum_{j \geq 0} t^{j}\left(\frac{1-q^{j+1}}{1-q}\right)^{n}
$$




$$
\begin{aligned}
& =\frac{1}{(1-q)^{n}} \sum_{j \geq 0} t^{j}\left(1-q^{j+1}\right)^{n}=\frac{1}{(1-q)^{n}} \sum_{j \geq 0} t^{j} \sum_{k=0}^{n}\left(\begin{array}{l}
n \\
k
\end{array}\right)(-1)^{k} q^{j k+k} \\
& =\frac{1}{(1-q)^{n}} \sum_{k=0}^{n}\left(\begin{array}{l}
n \\
k
\end{array}\right)(-1)^{k} q^{k} \sum_{j \geq 0}\left(t q^{k}\right)^{j}=\frac{1}{(1-q)^{n}} \sum_{k=0}^{n}\left(\begin{array}{l}
n \\
k
\end{array}\right) \frac{(-1)^{k} q^{k}}{1-t q^{k}},
\end{aligned}
$$

so that

$$
A_{n}(t, q)=\frac{1}{(1-q)^{n}} \sum_{k=0}^{n}\left(\begin{array}{l}
n \\
k
\end{array}\right)(-1)^{k} q^{k}(t ; q)_{k}\left(t q^{k+1} ; q\right)_{n-k}
$$

By examining (5.3) we can see that $A_{n}(t, q)$ is a polynomial in $t$ of degree at most equal to $(n-1)$, since the coefficient of $t^{n}$ in $A_{n}(t, q)$ must be equal to

$$
\begin{aligned}
-\frac{1}{(1-q)^{n}} \sum_{k=0}^{n}\left(\begin{array}{l}
n \\
k
\end{array}\right)(-1)^{k}(-1)^{n+1} q^{n(n+1) / 2} & \\
& =-\frac{(-1)^{n+1} q^{n(n+1) / 2}}{(1-q)^{n}} \sum_{k=0}^{n}\left(\begin{array}{l}
n \\
k
\end{array}\right)(-1)^{k}=0 .
\end{aligned}
$$

To see that $A_{n}(t, q)$ is a polynomial both in $t$ and $q$ we can use identity (5.3) and write

$$
\begin{aligned}
(1-q) A_{n}(t, q) & =\sum_{k=0}^{n}\left(\begin{array}{c}
n \\
k
\end{array}\right)(-1)^{k} q^{k} \frac{(t ; q)_{k}}{(1-q)^{n-1}}\left(t q^{k+1} ; q\right)_{n-k} ; \\
\left(1-t q^{n}\right) A_{n-1}(t, q) & =\sum_{k=0}^{n-1}\left(\begin{array}{c}
n-1 \\
k
\end{array}\right)(-1)^{k} q^{k} \frac{(t ; q)_{k}}{(1-q)^{n-1}}\left(t q^{k+1} ; q\right)_{n-k} .
\end{aligned}
$$

Hence

$$
\begin{aligned}
(1-q) & A_{n}(t, q)-\left(1-t q^{n}\right) A_{n-1}(t, q) \\
= & \sum_{k=1}^{n-1}\left(\begin{array}{c}
n-1 \\
k-1
\end{array}\right)(-q)^{k} \frac{(t ; q)_{k}}{(1-q)^{n-1}}\left(t q^{k+1} ; q\right)_{n-k}+(-q)^{n} \frac{(t ; q)_{n}}{(1-q)^{n-1}} \\
= & \sum_{j=0}^{n-2}\left(\begin{array}{c}
n-1 \\
j
\end{array}\right)(-q)^{j+1} \frac{(1-t)(t q ; q)_{j}}{(1-q)^{n-1}}\left(t q q^{j+1} ; q\right)_{n-1-j} \\
= & q(1-t)(-q)^{n-1} \frac{(t q ; q)_{n-1}}{(1-q)^{n-1}} \\
= & q(1-t) \sum_{j=0}^{n-1}\left(\begin{array}{c}
n-1 \\
j
\end{array}\right)(-q)^{j} \frac{(t q ; q)_{j}}{(1-q)^{n-1}}\left(t q q^{j+1} ; q\right)_{n-1-j}
\end{aligned}
$$

and consequently

$$
(1-q) A_{n}(t, q)=\left(1-t q^{n}\right) A_{n-1}(t, q)-q(1-t) A_{n-1}(t q, q) .
$$


With $A_{n}(t, q):=\sum_{j=0}^{n-1} t^{j} A_{n, j}(q)$ we deduce from (5.4) that the coefficients $A_{n, j}(q)$ satisfy the recurrence

$$
A_{n, j}(q)=[j+1]_{q} A_{n-1, j}(q)+q^{j}[n-j]_{q} A_{n-1, j-1}(q) .
$$

This shows that each polynomial $A_{n}(t, q)$ is a polynomial in $t, q$ with positive integral coefficients.

The first values of the polynomials $A_{n}(t, q)$ are reproduced in the following table:

$$
\begin{aligned}
A_{0}(t, q)= & A_{1}(t, q)=1 ; A_{2}(t, q)=1+t q ; A_{3}(t, q)=1+2 t q(q+1)+t^{2} q^{3} \\
A_{4}(t, q)= & 1+t q\left(3 q^{2}+5 q+3\right)+t^{2} q^{3}\left(3 q^{2}+5 q+3\right)+t^{3} q^{6} \\
A_{5}(t, q)= & 1+t q\left(4 q^{3}+9 q^{2}+9 q+4\right)+t^{2} q^{3}\left(6 q^{4}+16 q^{3}+22 q^{2}+16 q+6\right)+ \\
& t^{3} q^{6}\left(4 q^{3}+9 q^{2}+9 q+4\right)+t^{4} q^{10}
\end{aligned}
$$

Finally, from the identity $1 /(t ; q)_{n+1}=\sum_{j>0}\left[\begin{array}{c}n+j \\ n\end{array}\right]_{q} t^{j}$ we get

$$
\sum_{i=0}^{n-1} A_{n, i}(q) t^{i} \sum_{j \geq 0}\left[\begin{array}{c}
n+j \\
n
\end{array}\right]_{q} t^{j}=\sum_{k \geq 0}\left([k+1]_{q}\right)^{n} t^{k},
$$

and obtain for each $k$ the formula à la Worpitzky

$$
\sum_{i=0}^{n-1} A_{n, i}(q)\left[\begin{array}{c}
k+n-i \\
n
\end{array}\right]_{q}=\left([k+1]_{q}\right)^{n} .
$$

Surprisingly, it took another twenty years to Carlitz [Ca75] to construct a full combinatorial environment for the polynomials $A_{n}(t, q)$ he introduced in 1954.

\section{A detour to Combinatorics}

In contemporary combinatorics the following integral-valued statistics for permutations have been widely used. For each permutation $\sigma=$ $\sigma(1) \sigma(2) \cdots \sigma(n)$ of $12 \cdots n$ define:

$$
\begin{aligned}
\operatorname{exc} \sigma & :=\#\{i: 1 \leq i \leq n, \sigma(i)>i\} ; \\
\operatorname{des} \sigma & :=\#\{i: 1 \leq i \leq n-1, \sigma(i)>\sigma(i+1)\} ; \\
\operatorname{maj} \sigma & :=\sum_{\sigma(i)>\sigma(i+1)} i ; \\
\operatorname{inv} \sigma & :=\#\{(\sigma(i), \sigma(j)): i<j, \sigma(i)>\sigma(j)\} ;
\end{aligned}
$$

called number of excedances, number of descents, major index and inversion number, respectively. Those four statistics can also be defined for each permutation with repetitions ("multiset"). 
For $r \geq 1$ and each sequence $\mathbf{m}=\left(m_{1}, m_{2}, \ldots, m_{r}\right)$ of nonnegative integers let $R(\mathbf{m})$ denote the class of all $\left(\begin{array}{c}m_{1}+\cdots+m_{r} \\ m_{1}, \ldots, m_{r}\end{array}\right)$ permutations of the multiset $1^{m_{1}} 2^{m_{2}} \cdots r^{m_{r}}$. It was already known and proved by MacMahon [Mac15] that "exc" and "des," on the one hand, "maj" and "inv," on the other hand, were equidistributed on each class $R(\mathbf{m})$, accordingly on each symmetric group $\mathfrak{S}_{m}$.

However, we had to wait for Riordan [Ri58] for showing that if $A_{n, k}$ is defined to be the number of permutations $\sigma$ from $\mathfrak{S}_{n}$ having $k$ descents (i.e., such that $\operatorname{des} \sigma=k$ ), then $A_{n, k}$ satisfies recurrence (3.5):

$$
\begin{gathered}
A_{n, k}=(k+1) A_{n-1, k}+(n-k) A_{n-1, k-1} \quad(1 \leq k \leq n-1) ; \\
A_{n, 0}=1 \quad(n \geq 0) ; \quad A_{n, k}=0 \quad(k \geq n) .
\end{gathered}
$$

This result provides the following combinatorial interpretations for the Eulerian polynomials $A_{n}(t)=\sum_{\sigma \in \mathfrak{S}_{n}} t^{\operatorname{exc} \sigma}=\sum_{\sigma \in \mathfrak{S}_{n}} t^{\text {des } \sigma}$, the second equality being due in fact to MacMahon! The latter author [Mac15, p. 97, and p. 186] knew how to calculate the generating function for the classes $R(\mathbf{m})$ by "exc" by using his celebrated Master Theorem, but did not make the connection with the Eulerian polynomials. A thorough combinatorial study of those polynomials was made in the monograph [FS70] in 1970.

In 1974 Carlitz [Ca74] completes his study of his $q$-Eulerian polynomials by showing that

$$
A_{n}(t, q)=\sum_{\sigma \in \mathfrak{S}_{n}} t^{\operatorname{des} \sigma} q^{\operatorname{maj} \sigma} \quad(n \geq 0) .
$$

As "inv" has the same distribution over $\mathfrak{S}_{n}$ as "maj," it was very tantalizing to make a full statistical study of the pair (des, inv). Let $e_{q}(u):=\sum_{n \geq 0} u^{n} /(q ; q)_{n}$ be the (first) $q$-exponential. First, a straightforward calculation leads to the identity

$$
1+\sum_{n \geq 1} t A_{n}(t) \frac{u^{n}}{n !}=\frac{1-t}{1-t \exp ((1-t) u)} .
$$

In the above fraction make the substitution $\exp (u) \leftarrow e_{q}(u)$ and express the fraction thereby transformed as a $q$-series:

$$
\sum_{n \geq 0}{ }^{\mathrm{inv}} A_{n}(t, q) \frac{u^{n}}{(q ; q)_{n}}=\frac{1-t}{1-t e_{q}((1-t) u)} .
$$

The new coefficients ${ }^{\text {inv }} A_{n}(t, q)$ are to be determined. They were characterized by Stanley [St76] who proved the identity:

$$
{ }^{\operatorname{inv}} A_{n}(t, q)=t \sum_{\sigma \in \mathfrak{S}_{n}} t^{\operatorname{des} \sigma} q^{\operatorname{inv} \sigma} \quad(n \geq 1)
$$


Now rewrite the exponential generating function for the Eulerian polynomials $A_{n}(s)($ see $(3.1))$ as

$$
\sum_{n \geq 0} A_{n}(s) \frac{u^{n}}{n !}=\frac{(1-s) \exp u}{\exp (s u)-s \exp u}
$$

In the right-hand side make the substitutions $s \leftarrow s q$, $\exp (u) \leftarrow e_{q}(u)$. Again, express the fraction thereby transformed as a $q$-series:

$$
\sum_{n \geq 0}{ }^{\operatorname{exc}} A_{n}(s, q) \frac{u^{n}}{(q ; q)_{n}}=\frac{(1-s q) e_{q}(u)}{e_{q}(s q u)-s q e_{q}(u)}
$$

The combinatorial interpretation of the coefficients ${ }^{\text {exc }} A_{n}(s, q)$ was found by Shareshian and Wachs [SW07] in the form

$$
{ }^{\mathrm{exc}} A_{n}(s, q)=\sum_{\sigma \in \mathfrak{S}_{n}} s^{\operatorname{exc} \sigma} q^{\operatorname{maj} \sigma} \quad(n \geq 0)
$$

A further step can be made by calculating the exponential generating function for the polynomials $A_{n}(s, t, q):=\sum_{\sigma \in \mathfrak{S}_{n}} s^{\operatorname{exc} \sigma} t^{\operatorname{des} \sigma} q^{\operatorname{maj} \sigma} \quad(n \geq 0)$,
as was done in [FH08]:

$$
\sum_{n \geq 0} A_{n}(s, t, q) \frac{u^{n}}{(t ; q)_{n+1}}=\sum_{r \geq 0} t^{r} \frac{(1-s q)(u s q ; q)_{r}}{\left((u ; q)_{r}-s q(u s q ; q)_{r}\right)\left(1-u q^{r}\right)}
$$

Identities (4.2) that relate the evaluations of Eulerian polynomials at $t=-1$ to tangent numbers can also be carried over to a $q$-environment. This gives rise to a new family of $q$-analogs of tangent numbers using the combinatorial model of doubloons (see [FH09a], [FH09b].)

Following Reiner [Re95a, Re95b] Eulerian polynomials attached to other groups than the symmetric group have been defined and calculated, in particular for Weyl groups. What is needed is the concept of descent, which naturally occurs as soon as the notions of length and positive roots can be introduced. The Eulerian polynomials for Coxeter groups of spherical type have been explicitly calculated by Cohen [Co08], who gave the full answer to a question raised by Hirzebruch [Hi08], who, on the other hand, pointed out the relevance of Euler's memoir [Eul1768] to contemporary Algebraic Geometry.

Acknowledgements. The author is most thankful to Hyung Chan Jung (Sogang University), who kindly proofread the paper and corrected several typos. 


\section{DOMINIQUE FOATA}

\section{References}

[Be1713] Bernoulli, Jacques. Ars conjectandi, opus posthumum. Accedit Tractatus de seriebus infinitis, et epistola gallicé scripta de ludo pilae reticularis. Basileae: impensis Thurnisiorum, fratrum, 1713 .

[Ca54] Carlitz, Leonard. $q$-Bernoulli and Eulerian numbers, Trans. Amer. Math. Soc., vol. 76, 1954, p. 332-350.

[Ca75] Carlitz, Leonard. A combinatorial property of $q$-Eulerian numbers, Amer. Math. Monthly, vol. 82, 1975, p. 51-54.

[Co08] Cohen, Arjeh M. Eulerian polynomials of spherical type, Münster J. Math., vol. 1, 2008, p. 1-7.

[Eul1755] Euler, Leonhard. Institutiones calculi differentialis cum eius usu in analysi finitorum ac Doctrina serierum, Academiae Imperialis Scientiarum Petropolitanae, St. Petersbourg, 1755, chap. VII ("Methodus summandi superior ulterius promota").

[Eul1768] Euler, Leonhard. Remarques sur un beau rapport entre les séries des puissances tant directes que récriproques, Mémoires de l'Académie des Sciences de Berlin, vol. 27, 1768, p. 83-106. Also in Opera Omnia, Ser. I, Commentationes analyticae ad theoriam serierum infinitarum pertinentes, II, vol. 15, p. 70-90, Teubner, Leipzig, 1927 .

[FH08] Foata, Dominique; Han, Guo-Niu. Fix-Mahonian Calculus, III: A quadruple distribution, Monatshefte für Math., vol. 154, 2008, p. 177-197.

[FH09a] Foata, Dominique; Han, Guo-Niu. Doubloons and new $q$-tangent numbers, 16 p., to appear in Quarterly J. Math.

[FH09b] Foata, Dominique; Han, Guo-Niu. The doubloon polynomial triangle, 20 p., to appear in Ramanujan J. (The Andrews Festschrift).

[FS70] Foata, Dominique; Schützenberger, Marcel-Paul. Théorie géométrique des polynômes eulériens. Lecture Notes in Mathematics, 138, Berlin, Springer-Verlag, 1970. (http://igd.univ-lyon1.fr/ slc/books/index.html).

[Ge1852] Genocchi, Angelo. Intorno all'espressione generale de'numeri Bernulliani, Ann. Sci. Mat. Fis., vol. 3, 1852, p. 395-405.

[GR90] Gasper, George; Rahman, Mizan. Basic hypergeometric series. Encyclopedia of Math. and its Appl. 35, Cambridge Univ. Press, Cambridge, 1990.

[He1847] Heine, Heinrich Eduard. Über die Reihe..., J. reine angew. Math., vol. 34, 1847, p. $210-212$.

[Hi08] Hirzebruch, Friedrich. Eulerian Polynomials, Münster J. Math., vol. 1, 2008, p. 9-14.

[Mac15] MacMahon, Percy Alexander. Combinatory Analysis, vol. 1 and 2. Cambridge, Cambridge Univ. Press, 1915, (Reprinted by Chelsea, New York, 1955.

[Ni23] Nielsen, Niels. Traité élémentaire des nombres de Bernoulli. Gauthier-Villars, Paris, 1923 .

[Re95a] Reiner, Vic. The distribution of descents and length in a Coxeter group, Electronic J. Combinatorics, vol. 2, 1995, R25.

[Re95b] Reiner, Vic. Descents and one-dimensional characters for classical Weyl groups, Discrete Math., vol. 140, 1995, p. 129-140.

[Ri58] Riordan, John. An Introduction to Combinatorial Analysis. New York, J. Wiley, 1958.

[SW07] Shareshian, John; Wachs, Michelle L. $q$-Eulerian Polynomials: Excedance Number and Major Index, Electron. Res. Announc. Amer. Math. Soc., vol. 13, 2007, p. 3345.

[St76] Stanley, Richard P. Binomial posets, Möbius inversion, and permutation enumeration, J. Combin. Theory Ser. A, vol. 20, 1976, p. 336-356.

Dominique Foata

Institut Lothaire, 1 rue Murner,

F-67000 Strasbourg, France

foata@unistra.fr 